\title{
TRANSFORMING GROWTH FACTOR $\beta$ IN RELATION TO CARDIAC ALLOGRAFT VASCULOPATHY AFTER HEART TRANSPLANTATION
}

Tarek Aziz, FRCS

Philip Hasleton, FRCP(Path)

Alison Wynn Hann, $\mathrm{PhD}^{\mathrm{a}}$

Nizar Yonan, FRCS

Abdul Deiraniya, FRCS

Ian V. Hutchinson, $\mathrm{PhD}^{\mathrm{b}}$
Background: Cardiac allograft vasculopathy is a frequent sequel to cardiac transplantation, but the role of cytokines on the subsequent development of vasculopathy is still largely unknown.

Methods: We retrospectively studied 172 heart transplant recipients to investigate the relationship between the development of vasculopathy and various factors including the presence of transforming growth factor (TGF- $\beta$ ) in the graft. Endomyocardial biopsy specimens were stained with antibodies for TGF- $\beta$ and CD+68, and a TGF- $\beta$ staining score was derived. Vasculopathy was diagnosed by angiography and rejection was graded according to the International Society of Heart and Lung Transplantation classification. TGF$\beta_{1}$ genotype was determined by polymerase chain reaction analysis of DNA.

Results: After a mean follow-up period of $68 \pm 32$ months, the prevalence of significant vasculopathy was $52 \%$. The TGF- $\beta$ staining score was higher in patients with more severe vasculopathy $(95 \%$ confidence interval $=8.9-12.1)$ than in those who showed minimal or mild vasculopathy score changes of more than 7 (95\% confidence interval $=3.4-5.1), P=.0001$. TGF- $\beta$ expression correlated with the degree of vasculopathy $(r=0.73, P<.0007)$ during the study period. Risks for vasculopathy were recipient homozygous TGF- $\beta$ genotype, recurrent rejection, recipient history of ischemic heart disease, donor male sex, old donor age (years), and donor history of subarachnoid hemorrhage.

Conclusion: A strong association exists between the expression of TGF- $\beta$ in cardiac biopsy specimens and the development of vasculopathy. TGF- $\beta$ in the cardiac allograft is related to its genotype and to the number of rejection episodes. Strategies to down-regulate TGF- $\beta$ production might improve the outcome of cardiac allografts. (J Thorac Cardiovasc Surg 2000;119:700-8)
$\mathrm{C}$ ardiac allograft vasculopathy (CAV) contributes ignificantly to long-term morbidity in heart transplant recipients, and it is the commonest cause of death and retransplantation after 2 years. ${ }^{1} \mathrm{CAV}$ is an accelerated form of coronary artery disease character-

From the Cardiac Transplant Unit, Wythenshawe Hospital, University Department of Statistics, ${ }^{a}$ School of Biological Science, ${ }^{b}$ Manchester University, Manchester, United Kingdom.

Read at the Twenty-fifth Annual Meeting of The Western Thoracic Surgical Association, Olympic Valley (Lake Tahoe), Calif, June 23-26, 1999.

Received for publication June 29, 1999; revisions requested Aug 23, 1999; revisions received Oct 11, 1999; accepted for publication Nov 2, 1999.

Address for reprints: T. M. Aziz, FRCS, Division of Thoracic Surgery, Hairmyres Hospital, Eagleshame Rd, East Kilbride, Scotland, G75 8RG, United Kingdom.

Copyright (C) 2000 by The American Association for Thoracic Surgery.

$0022-5223 / 2000 \$ 12.00+0 \quad \mathbf{1 2 / 6 / 1 0 4 3 3 6}$

doi:10.1067/mtc.2000.104336 ized by rapidly progressive vascular myointimal hyperplasia leading to either diffuse, concentric, homogenous lesions spreading uniformly along the length of arteries or focal stenoses. ${ }^{2}$ Coronary angiography is the most widely used technique for detection of CAV because it provides clinically useful information regarding the prevalence and severity of coronary artery lesions.

Although CAV has been the subject of intense study, the relationship between the occurrence and severity of this disease and cytokines is still unknown. The production of growth factors in the allograft coronary lesions has been demonstrated by means of in situ hybridization techniques. ${ }^{3}$ Inflammatory cells, including macrophages, as well as platelets and endothelial cells play direct and indirect roles in cardiac allograft injury and repair. ${ }^{4}$ Evidence is accumulating that the cytokine network is involved in generating and maintaining graft atherosclerosis. ${ }^{5}$ 
The contribution of transforming growth factor (TGF- $\beta$ ) to the development of coronary artery disease in heart transplants is undefined. Increased expression of TGF- $\beta_{1}$ in human vascular stenotic lesions has been previously reported. ${ }^{6}$ In solid organ transplantation, TGF- $\beta$ has been implicated in the development of glomerulosclerosis mediated by its dual action of increasing deposition and decreasing degradation of the extracellular matrix. ${ }^{7}$

In the present study we have examined the involvement of TGF- $\beta$, in association with histologic, clinical, and genetic factors, in the generation of angiographically proven CAV after orthotopic heart transplantation.

\section{Methods}

This retrospective study analyzed the risk factors for development of coronary artery disease in heart transplant recipients. The study involves immunohistochemical assessment of the recipient's endomyocardial biopsy tissue in the absence of cellular rejection. As the majority of our recipients had cellular rejection during the first 18 months after transplantation, we restricted our analysis to recipients who survived at least 24 months after their operation.

Patients. Between 1987 and 1996, 208 orthotopic heart transplantations were performed at our center. A total of 175 orthotopic heart transplant recipients survived more than 2 years, and they were considered for this study. The age at transplantation was $46 \pm 10$ years and $81 \%$ were men. Recipients having heart retransplantation or those who survived for less than 24 months after the operation were excluded from this study. The standard technique ${ }^{8}$ was performed in 99 recipients and the bicaval ${ }^{9}$ procedure in 76 recipients. Follow-up was complete to December 1998 or to the time of death and ranged from 39 to 135 months.

Endomyocardial biopsies. Surveillance endomyocardial biopsies were performed through a percutaneous right internal jugular approach. At least 4 to 5 biopsies were performed during each biopsy session. The biopsy sessions were performed on a scheduled basis: weekly for the first month, every 2 weeks for the next 2 months, monthly until 6 months, then at 9,12 , and 18 months after transplantation, and yearly thereafter. In the event of clinical suspicion of rejection in the interim, further biopsies were performed as indicated. Biopsy specimens were evaluated for rejection by means of International Society for Heart and Lung Transplantation (ISHLT) criteria. ${ }^{10}$

Triple-drug immunosuppression with cyclosporine (INN: ciclosporin), azathioprine, and steroid therapy was used in all patients in addition to an initial 3-day application of cytolytic induction therapy (rabbit-antithymocyte globulin, 2 $\mathrm{mg} / \mathrm{kg}$ ). Acute rejection was treated with bolus methylprednisolone (500 mg daily for 3 days) and follow-up endomyocardial biopsy was performed 1 to 2 weeks later to assess outcome of treatment. Treatment was given only when the degree of rejection was $3 \mathrm{~A}$ or more or when the patients were in a clinically compromised condition. For the purpose of this study, a rejection episode was defined as the presence in at least one biopsy specimen of ISHLT grade 2 rejection or higher. Subsequent positive biopsy results were considered to be the same rejection episode if not separated by a rejectionfree biopsy result.

Coronary angiography and definition of graft atherosclerosis. Each patient underwent surveillance coronary angiography starting 2 years after transplantation and repeated annually at the time of biopsy.

Coronary angiograms were assessed by means of the protocol for angiographic assessment of coronary artery disease in heart transplant recipients as described previously by the Cardiac Transplant Research Database group. ${ }^{11}$ The degree of CAV was assessed in all the main coronary vessels and their primary branches. A primary coronary vessel was defined as the proximal two thirds of the left anterior descending artery, left circumflex, or a dominant or co-dominant right coronary artery. A branch vessel was defined as the distal one third of the left anterior descending artery, its diagonal branches, obtuse marginal branches, intermediate artery, and/or a nondominant right coronary artery. Scoring of $\mathrm{CAV}$ was based on involvement of the left main stem coronary artery, primary, and branch vessel stenoses. The disease score is shown in Table I. The final score was calculated for each patient and was assessed as no CAV (score $=0$ ), minimal CAV (score 1-3), mild CAV (score 4-7), moderate CAV (score 8-10), and severe CAV (score $\geq 10$ ).

Since donors were young (36 \pm 10 years) and recipients receiving an old donor heart ( $>50$ years) had a coronary angiogram soon after transplantation, we did not assume that early CAV represented pre-existing donor disease.

Histologic examination and immunohistochemistry. Only endomyocardial biopsy specimens taken at least 2 years after transplantation were selected for immunohistochemical assessment. A total of 4721 endomyocardial biopsy specimens, taken mainly from the interventricular septum obtained during baseline follow-up and at the time of angiographic studies, were graded according to the ISHLT classification system. ${ }^{10}$ So that the immediate effects of current rejection could be avoided, specimens taken from recipients in whom the previous biopsy result showed evidence of cellular rejection (any grade other than 0 ) were excluded from immunohistochemical staining for the purposes of this study $(n=863$ biopsy specimens). Also, specimens taken from patients requiring additional immunosuppression for any reason were also excluded ( $\mathrm{n}=199$ biopsy specimens). The rest of the endomyocardial biopsy specimens $(n=3659)$ were included for immunohistochemical staining.

Paraffin-embedded sections fixed in $4 \%$ formaldehyde were dewaxed and rehydated with limonene (Citroclear; HD Supplies, Aylesbury, Bucks, United Kingdom), alcohol, and water for 10 minutes and then treated with $10 \%$ proteinase $\mathrm{K}$ (Dako, Bucks, United Kingdom) in Tris-buffered saline (TBS) solution. Nonspecific binding was blocked with $10 \%$ normal swine serum (Chemicon International Ltd, Harrow, United Kingdom). Mouse anti-human TGF- $\beta$ antibody was 
Table I. Categorization of $C A V$

\begin{tabular}{lccc}
\hline & \multicolumn{3}{c}{ CAV score for each vessel stenosis } \\
\cline { 2 - 4 } Vessel stenosis & LMS & Primary vessel & Branches \\
\hline Normal & 0 & 0 & 0 \\
$<50 \%$ & 3 & 2 & 1 \\
$50 \%-70 \%$ & 6 & 4 & 2 \\
$70 \%-90 \%$ & 9 & 6 & 3 \\
$>90 \%$ & 12 & 8 & 4
\end{tabular}

The lesions were scored according to their severity and vessel significance. $L M S$, Left main stem coronary artery.

Table II. TGF- $\beta$ scoring system

\begin{tabular}{lc}
\hline Histopathologic assessment & Score \\
\hline Positive TGF- $\beta$ staining in cells & \\
$\quad$ Yes & 1 \\
No. of macrophages per high-power field & \\
$0-10$ & 0 \\
$11-19$ & 1 \\
$20-29$ & 2 \\
$>30$ & 3 \\
Degree of cellular staining & 1 \\
Light & 2 \\
Medium & 3 \\
Deep & \\
Positive TGF- $\beta$ staining in fibrous tissue & 1 \\
Yes & \\
Percent of fibrous tissue positive & 0 \\
$0 \%-4 \%$ & 1 \\
$5 \%-19 \%$ & 2 \\
$20 \%-49 \%$ & 3 \\
$>50 \%$ & \\
Degree of fibrous tissue staining & 1 \\
Light & 2 \\
Medium & 3 \\
Deep & 14 \\
Total maximum score & \\
\hline
\end{tabular}

diluted 1:10 in TBS and applied to 3 of the 4 sections on each slide while the remaining section received only TBS without antibodies. The slides were incubated for 1 hour, then washed and stained with peroxidase-conjugated anti-mouse immunoglobulin $\mathrm{G}$ at 1:1000 dilution (Sigma Immunochemical, St Louis, Mo) for 2 hours, after which slides were transferred to fresh diaminobenzidine. The slides were counterstained with Meyer's haemalum (Sigma Immunochemical), dehydrated, and mounted with dibutyl polystyrene xylene (DPX). To confirm the staining specificity, we performed blocking studies with recombinant TGF- $\beta$ (R\&D Systems, Abingdon, Oxon, United Kingdom) to inhibit binding of anti-TGF- $\beta$ antibodies. Macrophages were identified in sections by means of an indirect immunoperoxidase technique with a mouse monoclonal antibody, CD68 (reagent PGM-1, Dako).
Table III. Number of angiographic and immunohistochemistry assessments for the study population

\begin{tabular}{lc}
\hline No. of patients & Frequency of assessment \\
\hline 36 & $\geq 6$ \\
33 & 5 \\
30 & 4 \\
41 & 3 \\
19 & 2 \\
13 & 1 \\
\hline
\end{tabular}

\section{Immunohistochemistry quantification}

$T G F-\beta$ staining assessment. We used the same immunohistochemical staining score that was previously described by our laboratory for assessment of TGF staining in human allografts. ${ }^{12}$ The scoring for TGF- $\beta$ and CD+68 scoring took into account 3 factors:

1 . The absolute number of TGF- $\beta$ staining cells and CD68positive cells per high-power field $\left(0.202 \mathrm{~mm}^{2}\right)$ was quantified with an eyepiece graticule. At least 40 fields were counted with the use of a $\times 40$ objective lens so that the percentage standard deviation was less than $2 \%$ for at least 5 fields. The fields covered the entire field of each biopsy and any biopsy specimen without 40 separate fields was excluded.

2. The percentage and number of positive cells (myocytes, interstitium, or inflammatory cells) was also assessed by quantifying the number of cellular infiltrates and expressing them as a percentage of total number of infiltrating cells per field at $4 \times 40$ objective.

3 . The intensity of positive cellular staining was evaluated (Table II).

Fibrosis assessment. Fibrosis was assessed in sections stained with hematoxylin and eosin. Slides were scanned with a $\times 10$ objective lens. Each successive field was individually assessed for severity (as a percentage) of interstitial myocardial fibrosis (see Table II). After the entire section was examined, the mean score of all the fields was taken as the fibrosis score. Two independent blinded observers scored each biopsy specimen. A similar score was achieved in $81 \%$ of the biopsy specimens. Biopsy specimens with scoring differences of more than 1 point ( $n=703$ biopsy specimens) were reviewed to reach an agreement regarding the final score of the slides.

Each biopsy specimen consisted of 3 to 5 pieces. TGF score was calculated for each separate piece and then added together to calculate mean TGF- $\beta$ for each single biopsy specimen.

Development of TGF- $\beta$ and CAV scoring systems for each patient. Mean TGF- $\beta$ and CAV scores were calculated annually for each patient starting from 24 months after the operation till the end point of this study (patient death or December 1998). In total, 679 angiographic assessments were performed for the entire study population (Table III).

Genotyping of TGF- $\beta_{1}$ gene polymorphisms in transplant recipients. Patients were genotyped for a biallelic 
polymorphism in the TGF- $\beta_{1}$ gene related to higher or lower TGF- $\beta$ production in vitro. The 2 alleles encode either arginine at codon 25 , associated with higher TGF- $\beta$ production, or proline, which is associated with lower TGF- $\beta$ production. DNA was extracted from whole blood and genotyped by means of a polymerase chain reaction-based method. ${ }^{13}$

Data collection and statistical analysis. The mean followup period for the study population was $68 \pm 32$ months after heart transplantation. In addition to vasculopathy, the study investigators collected vasculopathy score, TGF score, and 16 demographic and medical history variables believed to be relevant to the development of CAV after heart transplantation. Medical history variables were defined as patient report of a previous diagnosis by a physician. All statistical analyses were completed with SPSS software (Windows 7.5; SPSS, Inc, Chicago, Ill). A univariate analysis was done that analyzed the possible relationship between CAV and the variables collected. Contingency table analyses for categorical data and $t$ tests or their nonparametric analog (Mann-Whitney $U$ test) for continuous variables were used. Some variables required $\log$ transformations to achieve approximate normality or constancy or additivity of scale. Any variable that achieved a $P$ value of .10 or less in the univariate analysis was included in the model. Multivariable analyses were performed by means of the Cox proportional hazard model on StatView J 4.11 software (Abacus Concepts, Inc, Berkeley, Calif). Forward and backward stepwise procedures were used to determine the combination of factors that were significant in the development of CAV after heart transplantation. If variables could be used as continuous variables, continuous variables were used in multivariate analysis. The regression and correlation analysis was used to compare the values of the immunohistochemistry score, TGF score, and the prevalence of rejection episodes. Adjusted correlation taking the time interval into consideration was used to assess the relation between TGF score and immunohistochemistry score during the study.

\section{Results}

Two years after transplantation (study beginning and baseline assessment). Thirty-four angiograms were performed within the first 18 months after transplantation because of graft dysfunction in 4, old donor age in 6 , and symptoms of congestive heart failure in 24 cases. Minimal to mild coronary artery disease was identified in the first year after heart transplantation in only 3 recipients, and they have been excluded from our analysis.

After the analysis of the surveillance recipients' angiograms at a 1-year interval after transplantation, we could classify the recipients into 4 groups: 131 (76\%) with normal-minimal coronary artery anatomy, $31(16.5 \%)$ with mild graft CAV, 6 (3\%) with moderate $\mathrm{CAV}$, and $8(4.5 \%)$ with severe CAV.
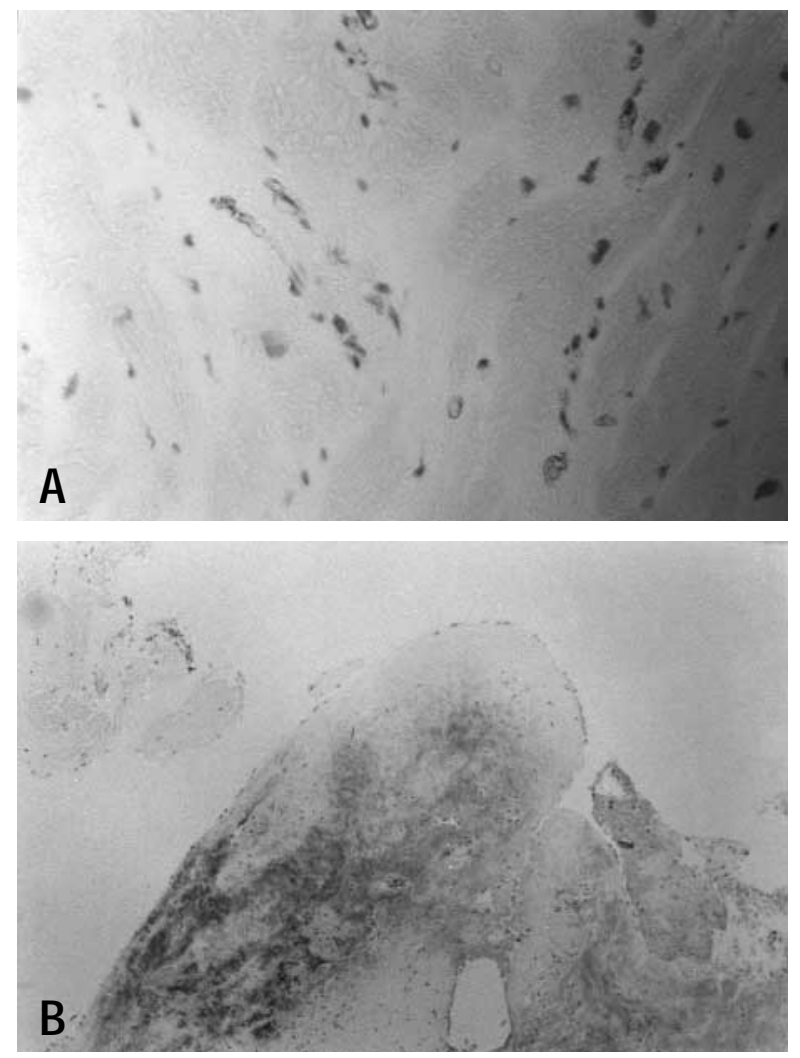

Fig 1. TGF- $\beta$ staining of the myocardial biopsy specimen. A, Low staining score in a specimen showing no or minimal CAV. B, Strong staining score in an example of severe CAV.

TGF- $\beta$ was immunolocalized in the myocardium, interstitium, blood vessels, macrophages, and in areas of fibrosis (Fig 1). TGF- $\beta$ was strongly expressed in the endomyocardial biopsy specimen from patients with moderate or severe CAV (95\% confidence interval [CI] $=7.8-9.3)$ in comparison with those with minimal or mild CAV (95\% CI $=2.9-4.2), P=.0002$.

Correlation between CAV and TGF- $\beta$ scoring during the study period. During the study period (19871998 or the patient's death), both TGF- $\beta$ score and coronary artery disease score were assessed annually for each surviving patient. The annual development of moderatesevere CAV (coronary artery disease score > 7) was 3.9\% in our recipients. The number of repeated assessments for the study population is shown in Table III.

Increased TGF- $\beta$ positive staining preceded the angiographic detection of CAV by 11 to 15 months. Eighty-two percent of patients ( $\mathrm{n}=61$ patients $)$ who had a TGF- $\beta$ staining score of more than 7 at any point of the study and normal angiograms at that time had significant CAV develop within 12 months. 


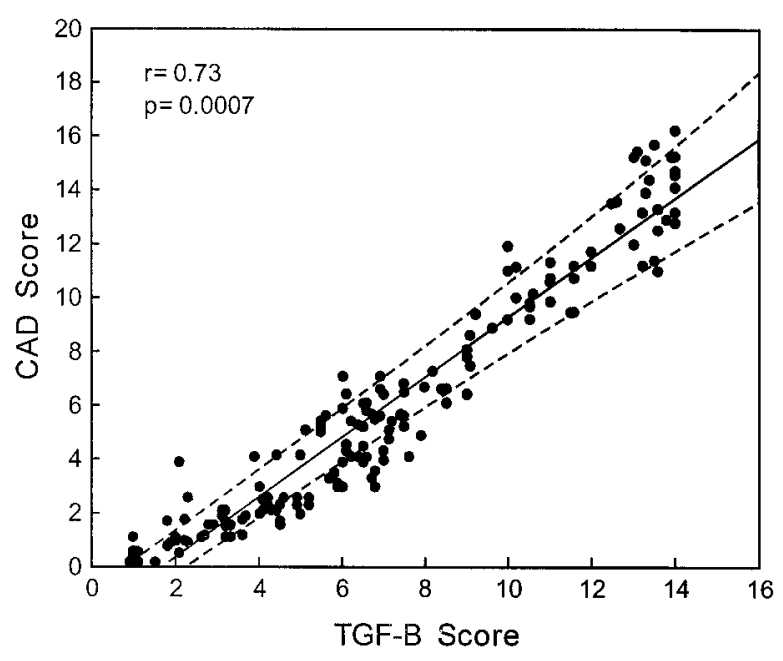

Fig 2. Correlation between TGF- $\beta$ staining score and the presence of $\mathrm{CAV}$ after transplantation.

Progression of allograft coronary artery disease was diagnosed in 76 patients with a TGF- $\beta$ score of more than 7 at an average of 12 months later. Progression was evidenced by new angiographic abnormalities in 69 patients and worsening of pre-existing mild-minimal coronary artery disease lesions in 7 patients.

New angiographic abnormalities were observed in 6 patients with mild coronary artery disease, in 32 patients with moderate disease, and in 31 patients with severe disease. These new abnormalities consisted mainly of new focal coronary artery disease (51 patients) and diffuse coronary artery disease (18 patients).

If the TGF- $\beta$ staining score was more than 10 , the likelihood of progression to severe coronary artery disease within 12 months was $41 \%$ compared with $15 \%$ when the TGF- $\beta$ score was less. Progression of coronary artery disease strongly correlated with a higher TGF- $\beta$ staining score in the endomyocardial biopsy specimens obtained during the same period $(r=0.73, P$ $=.0007)($ Fig 2).

Study end point. At the end of the study period (1998 or patient death), the recipients were classified into 4 groups according to their last angiographic assessment; 48 (28\%) with normal/minimal coronary artery anatomy, $34(19.5 \%)$ with mild graft CAV, 49 (28.5\%) with moderate CAV, and $41(24 \%)$ with severe graft CAV.

Higher TGF- $\beta$ expression was sustained in patients who had moderate or severe CAV (95\% CI $=8.9-12.1)$ compared with those who had minimal or mild CAV changes (score > 7; 95\% CI $=3.4-5.1$ ),$P=.0001$. Comparison of variables between recipients with mod- erate or severe CAV $(n=90)$ and those who did not show relevant CAV changes $(n=82)$ are summarized in Table IV.

Risk factors for CAV after transplantation and survival. The TGF- $\beta$ score at the study end point correlated with the number of rejection episodes that occurred during the first 2 years after transplantation $(r$ $=0.69, P=.003$ ) (Fig 3). Significant correlation has been also found between the prevalence of rejection episodes during the first 2 years after transplantation and the long-term development of graft CAV (Fig 4).

Multivariable analysis identified the weight of each variable in the development of coronary artery disease. TGF- $\beta$ score was the strongest determinant of disease development. Other significant factors were homozygous high TGF- $\beta$ producer genotype, number of grade 2 or more rejection episodes, donor age, recipient history of ischemic heart disease before transplantation, donor history of subarachnoid bleeding, and donor male sex (Table V).

Thirty-six patients have died, and the cause of death was reported to be moderate to severe graft atherosclerosis in 21 recipients. Among 15 (71\%) of 21 recipients who died of graft CAV, the final TGF score was more than 10. Multivariable analyses with the Cox proportional hazards model disclosed that TGF score was a single independent prognostic factors for survival in our study population (95\% CI 1.3-1.43 for each unit of the TGF score).

\section{Discussion}

Relevance of graft CAV after heart transplantation. CAV is the most common cause of morbidity and mortality in patients surviving for more than 2 years after transplantation, ${ }^{1,14}$ but the spectrum of lesions varies considerably. Since CAV is usually asymptomatic and sometimes develops and progresses rapidly, identification of patients at high risk is important.

Insights into pathology. The pathogenesis of CAV is poorly understood and is quite different from atherosclerosis in native coronary artery disease. Coronary arterial involvement is usually diffuse with long segments of all levels of vessels affected in a relatively uniform manner. It has been postulated that changes in the allograft coronary circulation originate from an interaction between immune and nonimmune factors that lead to smooth muscle cellular infiltration and accumulation of expanded neointima. Reape, Rayner, and Manning ${ }^{15}$ have reported excessive expression of cell adhesion molecules, chemokines, and increased numbers of macrophages in human atherosclerotic lesions. Labarrere, Nelson, and Faulk ${ }^{16}$ detected that 
Table IV. Comparison of variables between recipients according to their coronary artery disease status

\begin{tabular}{|c|c|c|c|}
\hline & No/minimal/mild $C A V$ & Moderate/severe $C A V$ & \\
\hline Variable & CAV score $\leq 6(n=82$ recipients $)$ & CAV score $\geq 7$ ( $n=90$ recipients $)$ & $\mathrm{P}$ value \\
\hline Recipient age (y) & $43 \pm 6.5$ & $49 \pm 9.6$ & .2 \\
\hline History of IHD & $49(54 \%)$ & $72(80 \%)$ & .002 \\
\hline History of PVD & $3(3 \%)$ & $4(5 \%)$ & .6 \\
\hline Recipient male sex & $67(82 \%)$ & $74(81 \%)$ & .9 \\
\hline Diabetes before surgery & $4(4 \%)$ & $3(4 \%)$ & .7 \\
\hline Cholesterol > $7 \mathrm{mmol} / \mathrm{L}$ & $4(4 \%)$ & $5(6 \%)$ & .5 \\
\hline Donor age (y) & $34 \pm 6.2$ & $43 \pm 8.2$ & .02 \\
\hline$<45 \mathrm{y}$ & $71(86 \%)$ & $57(63 \%)$ & .1 \\
\hline$>45 \mathrm{y}$ & $11(14 \%)$ & $33(37 \%)$ & .05 \\
\hline Donor sex & $46(56 \%)$ & $80(88 \%)$ & .004 \\
\hline SAH $\%$ of donors & $46(56 \%)$ & $69(76 \%)$ & .005 \\
\hline Ischemic time (min) & $196 \pm 44$ & $188 \pm 21$ & .3 \\
\hline Bypass time (min) & $261 \pm 31$ & $252 \pm 36$ & .6 \\
\hline Rejection episodes grade $\geq 2$ & $3.2 \pm 1.2$ & $6.0 \pm 1.8$ & .002 \\
\hline Obesity $\geq 20$ BMI & $2(3 \%)$ & $5(6 \%)$ & .2 \\
\hline TGF- $\beta$ score & $4.2 \pm 0.9$ & $10.3 \pm 1.6$ & .0003 \\
\hline Average macrophages/biopsy & $16 \pm 8$ & $39 \pm 13$ & .002 \\
\hline Homozygous high TGF- $\beta$ genotype & $66(80 \%)$ & $85(95 \%)$ & .03 \\
\hline Average CyA trough level at $2 \mathrm{y}$ & $121 \pm 26$ & $163 \pm 19$ & .04 \\
\hline Serum creatinine $>200 \mathrm{mmol} / \mathrm{L}$ & $11(13 \%)$ & $8(9 \%)$ & 6 \\
\hline
\end{tabular}

$I H D$, Ischemic heart disease; $P V D$, peripheral vascular disease; $B M I$, body mass index; $S A H$, subarachnoid hemorrhage; $C y A$, cyclosporine level.

early activation of coronary endothelial lining in the transplanted heart predicts the development of CAV. Other studies ${ }^{17}$ have shown infiltration of macrophages in chronic liver and renal allograft rejection. Consequent activation of endothelial and smooth muscle cells with subsequent expression of leukocyte activation molecules and increased secretion of adhesion molecules (P-selectin and intercellular adhesion molecule-1) may contribute to the development of CAV. ${ }^{18}$ These probably lead to endothelial surface changes previously identified as early events in the pathogenesis of acute transplant rejection and the subsequent development of CAV. With time, progressive myocardial fibrosis results and adventitial fibrosis of the subepicardial tissues probably inhibits dilatation and remodeling of the epicardial arteries by constriction. ${ }^{19}$

The role of TGF- $\beta$. TGF- $\beta$ modulates a number of crucial events potentially central to the genesis and maintenance of CAV. These include macrophage chemotaxis, suppression of lymphocyte function, fibroblast chemotaxis, and proliferation, in addition to the modulation of collagen synthesis. ${ }^{20,21}$ TGF- $\beta$ is also a strong stimulator of extracellular matrix synthesis. ${ }^{22}$ Many different cells can synthesize and release TGF- $\beta$, including activated macrophages, lymphocytes, and platelets $^{23,24}$ (cell types recruited intensely to sites of vascular injury in the development of the atherosclerotic process). ${ }^{22}$ Previous reports have confirmed increased expression of TGF- $\beta$ in vascular stenotic lesions. In addition to its stimulating effect on cellular and vascular smooth muscle proliferation, TGF- $\beta$ is also a strong stimulant of the secretion of endothelin, a potent arterial vasoconstrictor. ${ }^{25}$ In renal allograft recipients, TGF- $\beta$ has been shown to strongly correlate with the development of glomerulosclerosis. ${ }^{7}$

Gayle and associates ${ }^{26}$ assessed CAV on the basis of an evaluation of ischemic changes in the endomyocardial biopsy specimen. They concluded that ischemic myocardial changes in the biopsy specimen were highly specific for CAV (98\%), with a positive and negative predictive value for ischemic injury of $92 \%$ and $51 \%$, respectively. Their study also reported a low sensitivity for the presence of disease. An association between allograft rejection, the subsequent development of $\mathrm{CAV}$, and rejection episodes (especially those that are mild and untreated) has been reported. ${ }^{27,28}$

Current study. In the present study we have evaluated the risk factors for the development of CAV in cardiac allograft recipients at our center. We have demonstrated the significance of cardiac allograft cytokine expression and an association with the subsequent development of CAV. Recipients homozygous for high TGF- $\beta$ producer genotype were most likely to have high TGF- $\beta$ tissue expression. The level of TGF- $\beta$ expression in the endomyocardial biopsy specimen was the most potent predictor of disease. The number of 


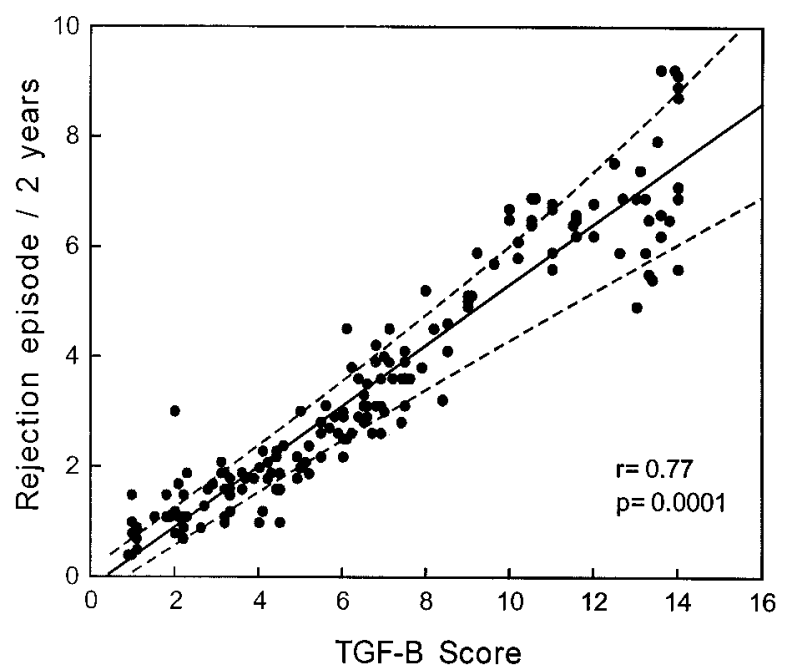

Fig 3. Correlation between TGF- $\beta$ staining score and number of rejection episodes of grade 2 or more during the first 2 years after transplantation. $C A D$, Coronary artery disease.

Table V. Multivariable analysis for risk factors for development of CAV after transplantation

\begin{tabular}{lccc}
\hline Variable & Risk factor & $95 \%$ CI & P value \\
\hline TGF- $\beta$ staining score/unit & 2.63 & $1.89-3.18$ & .0002 \\
Homozygous TGF- $\beta$ genotype & 1.59 & $1.33-1.73$ & .001 \\
Donor male sex & 1.19 & $1.12-1.24$ & .02 \\
Donor age > 45 y & 1.33 & $1-2.3$ & .03 \\
Rejection episode & 1.95 & $1.62-2.31$ & .003 \\
IHD before transplant & 1.66 & $1.2-1.92$ & .03 \\
Donor SAH & 1.11 & $1.02-1.21$ & .04 \\
\hline
\end{tabular}

$I H D$, Ischemic heart disease; $S A H$, subarachnoid hemorrhage.

ISHLT rejection episodes of grade 2 or more correlated significantly with higher myocardial TGF- $\beta$ deposition and the development of CAV.

These findings suggest that frequent cellular rejection episodes during the first 2 post-transplant years in recipients genetically predisposed to higher TGF- $\beta$ production initiate a series of inflammatory and immunologic responses characterized by overexpression of TGF- $\beta$. This culminates in endothelial injury, coronary intimal thickening, and development of CAV. In agreement with previous work, ${ }^{25,29}$ we also report a greater risk of the development of CAV in recipients with a history of ischemic heart disease and in those in whom the allograft donor was male. The increased incidence of CAV among recipients in whom the organ donor died of subarachnoid bleeding may be attributable to the fact that many of these donors are likely to have had chronic systemic hypertension.

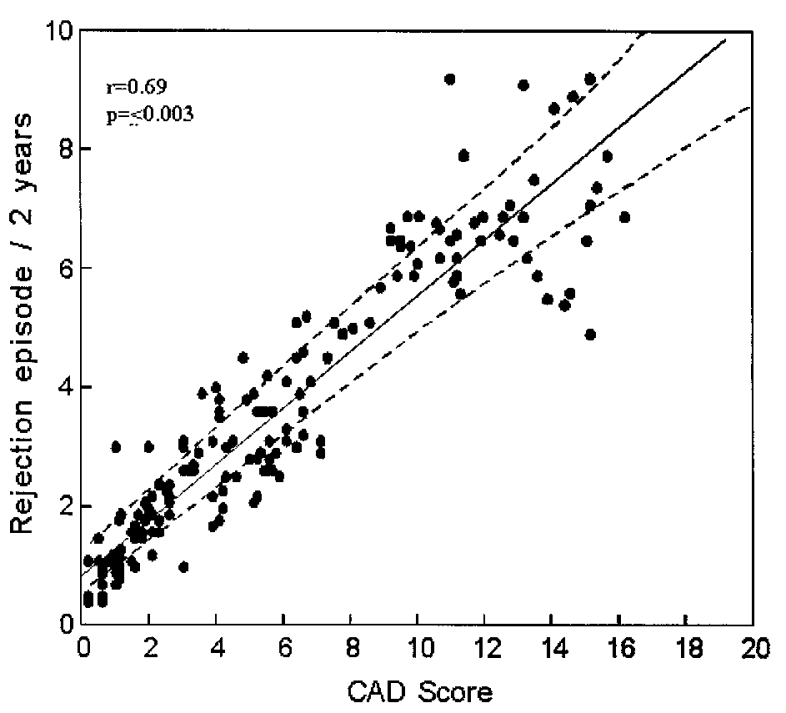

Fig 4. Correlation between TGF- $\beta$ staining score at the study end point and the number of rejection episodes of grade 2 or more during the first 2 years after transplantation. $C A D$, Coronary artery disease.

Study limitations. Our analysis was limited by the fact that the study population was restricted to recipients surviving for at least 2 years after transplantation and had undergone coronary angiography. We were unable, therefore, to provide information about the very early development of fatal CAV. The rationale for this approach was based on the requirement to avoid acute allograft rejection, particularly given the poor reliability of immunohistochemical staining during rejection (170 recipients had one or more episode of rejection during the first 2 years). However, in view of the relatively young donor age ( $32 \pm 9$ years), the incidence of donor CAV was likely to have been low in this population. Another limitation of our study was the suboptimal sensitivity of contrast coronary angiography for the detection of early CAV. The accuracy of coronary angiography for detection of nonepicardial coronary lesions is doubtful, and previous reports have demonstrated severe CAV at autopsy in transplant recipients shortly after a reportedly normal angiogram..$^{30,31}$

\section{Conclusions}

Despite these limitations, this study has highlighted the potential role of TGF- $\beta$ in the development of CAV for a period of up to 10 years after transplantation. The donor-related risk factors for CAV are also significant. Future studies at our institution will include the use of intracoronary vascular ultrasound in the detection of changes in different layers of the coronary artery and correlation with TGF- $\beta$ staining score in all surviving 
recipients. Increased understanding of the etiology of $\mathrm{CAV}$ and the role of cytokines in its development will allow a clearer definition of the group of patients at higher risk. We suggest that the management of CAV in heart transplant recipients can be considered in a stepwise fashion. First, individuals identified by their risk factors (Table V) should be monitored more closely. We suggest that high-risk patients be targeted for intracoronary vascular ultrasound even in the absence of angiographic evidence of CAV. Second, modification of the biopsy regimen and immunosuppression may be important for some patients, particularly those who are homozygous for TGF- $\beta_{1}$ gene. Third, a number of pharmacologic agents (eg, statins, antihypertensives), as well as better control of weight, diabetes, and hypertension, may have the potential to minimize or slow the occurrence of $\mathrm{CAV}$ in selected high-risk recipients. Finally, we suggest that immunologic strategies to manipulate cytokine expression in cardiac allografts may improve the survival and function of cardiac allografts.

\section{REFERENCES}

1. Hosenpud JD, Bennett LE, Keck BM, Fiol B, Boucek MM, Novick RJ. The Registry of the International Society for Heart and Lung Transplantation. J Heart Lung 1998;17:665-78.

2. Neiish AS, Loh E, Schoen FJ. Myocardial changes in cardiactransplant associated atherosclerosis: potential for timely diagnosis. J Am Coll Cardiol 1992;19:586-92.

3. Conraads V, Lahaye I, Rademakers F, et al. Cardiac allograft vasculopathy: aetiologic factors and therapeutic approach. Acta Cardiol 1998;53:37-43.

4. Ravalli S, Szabolcs M, Albala A, Michler RE, Cannon PJ. Increased immunoreactive endothelin-1 in human transplant coronary artery disease. Circulation 1996;94:2096-102.

5. Koskinen PK, Lemstorm KB. Adhesion molecule P-selectin and vascular cell adhesion molecule-1 in enhanced heart allograft arteriosclerosis in the rat. Circulation 1997;95:191-6.

6. Nikol S, Isner JM, Pickering JC, Kearney M, Leclerc G, Weir L. Expression of transforming growth factor beta 1 is increased in human vascular restenotic lesions. J Clin Invest 1992;90:158292.

7. Shihab FS, Andoh TF, Tanner AM, et al. Role of transforming growth factor-beta 1 in experimental chronic cyclosporine nephropathy. Kidney Int 1996;49:1141-51.

8. Lower RR, Stofer RC, Shumway NE. Homovital transplantation of the heart. J Thorac Cardiovasc Surg 1961;41:196.

9. Sarsam MA, Campbell CS, Yonan NA, Deiraniya AK, Rahman AN. An alternative technique in orthotopic cardiac transplantation. J Card Surg 1993;8:344-9.

10. Billingham ME, Cary NRB, Hammond ME, et al. A working formulation for the standardization of nomenclature in the diagnosis of heart and lung rejection: heart rejection study group. J Heart Transplant 1990;9:587-93.

11. Costanzo MR, Naftel DC, Pritzker MR, et al. Heart transplant coronary artery disease detected by angiography: a multiinstitutional study of operative donor and recipient risk factors. Cardiac Transplant Research Database. J Heart Lung Transplant 1998;17: 744-53.
12. El-Gamel A, Awad ME, Sim E, et al. Transforming growth factor-beta 1 and lung allograft fibrosis. Eur J Cardiothorac Surg 1998;4:424-30.

13. Perrey C, Pravica V, Sinnott PJ, Hutchinson IV. Genotyping for polymorphisms in interferon-gamma, interleukin-10, transforming growth factor-beta 1 and tumour necrosis factor-alpha genes: a technical report. Transplant Immunol 1998;63:193-7.

14. Graham AR. Autopsy finding in cardiac transplant patients: a 10year experience. Am J Clin Pathol 1992;97:369-75.

15. Reape TJ, Rayner K, Manning CD. Expression and cellular localization of the CC chemokines PARC and ELC in human atherosclerotic plaques. Am J Pathol 1999;145:365-74.

16. Labarrere CA, Nelson DR, Faulk WP. Endothelial activation and development of coronary artery disease in transplanted hearts. JAMA 1997;278:1169-75.

17. Sosroseno W, Herminajeng E. The immunoregulatory roles of transforming growth factor beta. Br J Biomed Sci 1995;52:142-8.

18. Koskinen PK, Lemstrom KB. Adhesion molecules P-selectin and vascular cell adhesion molecule-1 in enhanced heart allograft arteriosclerosis in the rate. Circulation 1997;95:191-6.

19. Lim TT, Liang DH, Botas J, Schroeder JS, Oesterle SN, Yeung AC. Role of compensatory enlargement and shrinking in transplant coronary artery disease: serial intravascular ultrasound study. Circulation 1997;95:855-9.

20. Moses HL, Yang EY, Pietenpol JA. Regulation of epithelial proliferation by TGF-beta. Ciba Foundation Symposium 1991;157: 66-74; discussion 75-80.

21. Moses HL. TGF-beta regulation of epithelial cells proliferation. Mol Prod Devel 1992;32:179-83.

22. Sosroseno W, Herminajeng E. The immunoregulatory role of transforming growth factor beta. $\mathrm{Br} \mathrm{J}$ Biomed Sci 1995;52:142-8.

23. Sporn MB, Roberts AB, Wakefield LM, Glick AB, Danielpour D. Transforming growth factor-beta and suppression of carcinogenesis. Princess Takamatsu Symposia 1989;20:259-66.

24. Sporn MB, Roberts AB. TGF-beta: problems and prospects. Cell Regulation 1990;1:875-82;48:959-66.

25. Maemura K, Kurihara H, Morita T, Oh-Hashi Y, Yazaki Y. Production of endothelin-1 in vascular endothelial cells is regulated by factors associated with vascular injury. Gerontology 1992;38(Suppl 1):29-35.

26. Gayle L, Winters MD, Frederick J, Schoen MD. Graft arteriosclerosis-induced myocardial pathology in heart transplant recipients: predictive value of endomyocardial biopsy. J Heart Lung Transplant 1997;16:985-93.

27. Costanzo-Nordin MR Cardiac allograft vasculopathy: relationship with acute cellular rejection and histocompatibility. J Heart Lung Transplant 1992;11:S90-103.

28. Mehra MR, Vetura HO, Chambers R, et al. Predictive model to assess risk for cardiac allograft vasculopathy: an intravascular ultrasound study. J Am Coll Cardiol 1995;26:1537-44.

29. Tanaka H, Sukhova GK, Swanson SJ, Cybulsky MI, Schoen FJ, Libby P. Endothelial and smooth muscle cells express leukocyte adhesion molecules heterogeneously during acute rejection of rabbit cardiac allografts. Am J Pathol 1994;144:938-51.

30. Dressler FA, Miller LW. Necropsy versus angiography: How accurate is angiography? J Heart Lung Transplant 1992;11:556-9.

31. Uretsky BF, Kormos RL, Zerb TR, et al. Cardiac events after heart transplantation: incidence and predictive value of coronary angiography. J Heart Lung Transplant 1992;11:S45-51. 


\section{Discussion}

Dr Leonard Bailey (Loma Linda, Calif). Dr Hutchinson, I applaud your effort to shed some new and relevant light on the still-perplexing issue of CAV. Your data suggest that 9 variables significantly separated the 102 recipients with trivial or no CAV from the 70 recipients who had angiographically documented moderate to severe CAV. Seven of these 9 variables, including your newly devised TGF- $\beta$ scoring system, retained significance in multiple regression analysis as independent risk factors for the development of coronary artery disease. Two most significant risk factors in your study were total number of rejection episodes and a TGF- $\beta$ score higher than 7.

I have a couple of comments to make, not to mention the insensitivity of routine angiography to identify significant degrees of CAV.

The first comment relates to the details of the TGF- $\beta$ scoring system. About half the points contributing to the score seemed reasonably quantitative and hence objective in nature. They include the number of macrophages and the percent fibrosis in the biopsy specimens. The remainder of the scoring system seems quite subjective, based on shades of staining or presence of TGF- $\beta$. I have two questions relating to that. First, were the histologic graders blinded to the study group data?

Dr Hutchinson. Yes, they were.

Dr Bailey. Second, your database included more than 12,000 myocardial biopsy specimens. How many were actually subjected to immunohistochemical analysis for the purposes of this study?

Dr Hutchinson. About 3500.

Dr Bailey. About a fourth of them. That is a lot of work. My second comment relates to the role of TGF- $\beta$ and solid organ transplantation. TGF- $\beta$ is a multifunctional protein among a large family of cytokines that play a role in the repair of vessel injuries. It is, of course, of enormous interest to our interventional cardiology colleagues since their balloons and stents seem to up-regulate cell growth and proliferative functions of this molecule. However, TGF- $\beta$ is also a powerful immunoregulator, and its immunosuppressive behavior or function is currently under investigation as a transgene in experimental studies. You have suggested immunologic down-regulation of TGF- $\beta$ as a strategy to reduce CAV. Since TGF- $\beta$ is so ubiquitous in recipient tissue and its global effects on cell growth and differentiation are so important to the health of the recipient, would it not be better to leave it alone, simply as a marker of graft injury or in this case as a possible prognostic indicator for the development of coronary vascular disease? It occurred to me alternatively that you could examine methods of up-regulating the immunosuppressive functions of this molecule to enhance graft survival. Could you comment on any of those issues?
Dr Hutchinson. TGF- $\beta$ has many functions, including suppression of lymphocyte proliferation. In the acute situation, a little bit of TGF is probably beneficial. However, in the chronic situation in which TGF is up-regulated, chronic rejection of the lung, kidney or liver is likely to develop in patients who are genetically inclined to overproduction of TGF. As an example, 38 of 39 individuals in whom $80 \%$ restenosis developed are the high TGF- $\beta$ genotype. That is ample evidence that TGF is associated with the kind of lesion that develops in the transplant. TGF is not the only factor involved. We are currently investigating the genetics of vascular endothelial growth factor, platelet-derived growth factor, $\alpha$ and $\beta$, basic fibroblast growth factor, and insulin-like growth factor-1. All of those may play a role, but the predominant one appears to be TGF- $\beta$. In the chronic situation, TGF- $\beta$ appears to be playing a major role in the development of disease.

Dr Bailey. How do you suggest we in the transplant community use this information in the day-to-day surveillance of our patients?

Dr Hutchinson. I think you can look at biopsy specimens very easily for staining for TGF, regardless of whether you want to develop a very complex scoring system, as our pathologists have. Our pathologists were blinded, and each slide was read by at least two or three of them. They conferred to agree on a score, and they used all sorts of controls for the intensity of staining. Our pathologists exercised a great deal of care, but TGF- $\beta$ staining could be incorporated into a routine pathologic system.

You mentioned immunosuppression. We know that cyclosporine increases TGF- $\beta$ production both in vivo and in vitro, and there is actually a very close correlation between cyclosporine trough levels and circulating levels of TGF. Also, at least in lung transplant recipients, there is an association between the development of lung fibrosis and the cyclosporine trough level. We might actually think about switching our longer term patients from cyclosporine to something less damaging.

Dr Mark Pelletier (Montreal, Quebec, Canada). I have a question about TGF- $\beta$, one of its roles being that of a proangiogenic factor. In these patients with severe $\mathrm{CAV}$, might the expression of TGF- $\beta$ in fact be secondary to the heart's need to make new vessels, perhaps new collateral vessels? Would TGF- $\beta$ be increased in the same manner as vascular endothelial growth factor or basic fibroblast growth factor? Have you considered this possibility?

Dr Hutchinson. It appears to be the other way around, because the genotype predicts the development of coronary artery disease, rather than the other way around. However, I think you are right about vascular endothelial growth factor, and we are looking at the genotype associated with that. 